\title{
Associations between twelve common gene polymorphisms and susceptibility to hepatocellular carcinoma: evidence from a meta-analysis
}

\author{
Yi Quan ${ }^{1}$, Jun Yang ${ }^{1}$, Tao Qin ${ }^{1}$ and Yufang $\mathrm{Hu}^{2^{*}}$
}

\begin{abstract}
Background: Associations between polymorphisms in vitamin D receptor (VDR)/vascular endothelial growth factor (VEGF)/interleukin-18 (IL-18)/mannose-binding lectin (MBL) and susceptibility to hepatocellular carcinoma (HCC) were already explored by many studies, yet the results of these studies were inconsistent. The aim of this metaanalysis was to better clarify associations between polymorphisms in VDRNEGF/L-18/MBL and HCC by combing the results of all relevant studies.

Methods: Eligible publications were searched from PubMed, Embase, WOS, and CNKI. We used Review Manager to combine the results of individual studies.

Results: Thirty studies were included in this study. Combined results revealed that VDR rs7975232, VDR rs2228570, VEGF rs699947, VEGF rs3025039, IL-18 rs1946518, and MBL rs7096206 polymorphisms were all significantly associated with HCC in the overall pooled population. We also obtained similar significant associations for VDR rs7975232, VDR rs2228570, IL-18 rs1946518, and MBL rs7096206 polymorphisms in Asians.

Conclusions: Collectively, this meta-analysis proved that VDR rs7975232, VDR rs2228570, VEGF rs699947, VEGF rs3025039, IL-18 rs1946518, and MBL rs7096206 polymorphisms may confer susceptibility to HCC in certain populations.
\end{abstract}

Keywords: Vitamin D receptor (VDR), Vascular endothelial growth factor (VEGF), Mannose-binding lectin (MBL), Interleukin-18 (IL-18), Hepatocellular carcinoma (HCC), Meta-analysis

\section{Background}

Hepatocellular carcinoma (HCC) is one of the leading causes of death all over the world [1,2]. Although we still did not reveal the exact mechanism of its pathogenesis, it was evident that genetic components were essential in the development of HCC. Firstly, the incidences of HCC in different populations were quite different $[3,4]$, and genetic background was probably one of the reasons behind differences in disease prevalence across different populations. Secondly, numerous susceptible genetic loci of HCC

\footnotetext{
* Correspondence: huyfangh@163.com

2Department of Radiology, Affiliated Hospital of Guilin Medical University, No.

15 of Lequn Road, Guilin 540001, Guangxi, China

Full list of author information is available at the end of the article
}

were also identified and validated by existing genetic association studies $[5,6]$.

Mannose-binding lectin (MBL) and interleukin-18 (IL-18) are crucial modulators of immunological reactions, whereas vitamin $\mathrm{D}$ receptor (VDR) and vascular endothelial growth factor (VEGF) are vital for both immune-regulation and angiogenesis [7-10]. So, if a genetic polymorphism could alter the transcription activity of $V D R / V E G F / I L-18 / M B L$ or the protein structure of VDR/VEGF/IL-18/MBL, there is a possibility that this polymorphism may lead to the development of chronic inflammatory cellular injuries and also confer susceptibility to many types of malignancy including HCC.

(C) The Author(s). 2019 Open Access This article is distributed under the terms of the Creative Commons Attribution 4.0 International License (http://creativecommons.org/licenses/by/4.0/), which permits unrestricted use, distribution, and reproduction in any medium, provided you give appropriate credit to the original author(s) and the source, provide a link to the Creative Commons license, and indicate if changes were made. The Creative Commons Public Domain Dedication waiver (http://creativecommons.org/publicdomain/zero/1.0/) applies to the data made available in this article, unless otherwise stated. 
In the past 20 years, many studies explored associations between polymorphisms in VDR/VEGF/IL-18/MBL and $\mathrm{HCC}$, yet the conclusions of these studies were somehow inconsistent [11-40]. To better clarify associations between polymorphisms in VDR/VEGF/IL-18/MBL and $\mathrm{HCC}$, we designed this study to get a more credible conclusion by combing the results of all relevant studies.

\section{Methods}

We wrote this meta-analysis in accordance with the requirements of the PRISMA guideline [41].

\section{Literature search and inclusion criteria}

To retrieve eligible articles, we searched PubMed, WOS, Embase, and CNKI with keywords listed below: ("vitamin D receptor" or "VDR" or "vascular endothelial growth factor" or "VEGF" or "interleukin 18" or "IL 18" or "mannose-binding lectin" or "Mannose-binding protein" or "MBL" or "MBP") and ("polymorphism" or "variant" or "variation" or "mutation" or "SNP" or "genome-wide association study" or "genetic association study" or "genotype" or "allele") and ("hepatocellular carcinoma" or "HCC"). The references of retrieved articles were also screened by us to identify other potentially relevant articles.

To be included in this meta-analysis, some criteria must be met: (I) about associations between polymorphisms in $V D R / V E G F / I L-18 / M B L$ and HCC in humans; (II) Offer genotypic distribution of VDR/VEGF/IL-18/MBL polymorphisms in patients with HCC and controls; (III) full manuscript in English or Chinese is retrievable. Publications were deemed to be ineligible for inclusion if (I) not about polymorphisms in VDR/VEGF/IL-18/MBL and HCC; (II) narrative reviews, systematic reviews, or comments; (III) studies only involved HCC patients. We only included the most up to date study for analyses if duplicate publications were found during the literature search.

\section{Data extraction and quality assessment}

Two authors extracted the following essential information from eligible studies: (I) name of the leading author; (II) published year; (III) country of the leading author; (IV) ethnicity of involved participants; (V) number of patients with HCC and controls in each study; (VI) genotype distributions of polymorphisms in VDR/VEGF/ $I L-18 / M B L$ among patients with HCC and controls. $P$ values of Hardy-Weinberg equilibrium (HWE) were also calculated.

The authors used the Newcastle-Ottawa scale (NOS) to assess the quality of eligible publications [42]. The score range of NOS is between 0 and 9, when a study got a score of 7 or more, we considered that the methodology quality of this study was good

Two authors extracted data and assessed the quality of eligible studies. The authors wrote to the leadings authors for additional information if essential information was found to be incomplete.

\section{Statistical analyses}

We used Review Manager to combine the results of individual studies. $Z$ test was employed to assess associations between polymorphisms in VDR/VEGF/IL-18/MBL and susceptibility to HCC. The statistical significance threshold of $P$ value was set at 0.05 . We used $I^{2}$ statistics to assess between-study heterogeneities. We used Randomeffect models (DerSimonian-Laird method) to combine the results if $I^{2}$ is larger than 50\%. Otherwise, fixedeffect models (Mantel-Haenszel method) were used to combine the results $[43,44]$. We further carried out subgroup analyses by ethnicity to get ethnic-specific results. We examined the stability of combined results by deleting one study each time and combining the results of the remaining studies. We used funnel plots to estimate whether our combined results may be influenced by publication biases.

\section{Results}

\section{Characteristics of included studies}

We found 168 articles during literature searching. Fortyfive articles were assessed for eligibility after excluding unrelated or duplicate articles. We further excluded eight reviews and six case series, and another one publication was excluded because of missing crucial data. Totally, 30 articles were ultimately found to be eligible for inclusion (Fig. 1). Extracted data of eligible articles were summarized in Table 1.

Meta-analyses results for polymorphisms in VDR and HCC Six studies were eligible for estimation of associations between polymorphisms in VDR and HCC. VDR rs7975232 (dominant comparison OR $=1.58,95 \% \mathrm{CI}$ 1.04-2.39; over-dominant comparison $\mathrm{OR}=0.80,95 \%$ CI 0.65-0.98) and rs2228570 (dominant comparison OR $=1.54,95 \%$ CI 1.25-1.89; recessive comparison OR = 0.67, $95 \%$ CI 0.54-0.84; allele comparison OR $=1.34$, 95\% CI 1.06-1.68) polymorphisms were found to be significantly associated with HCC in overall combined analyses. Subgroup analyses showed similar positive findings for rs7975232 (dominant comparison) and rs2228570 (dominant, recessive, and allele comparisons) polymorphisms in East Asians (see Table 2 and Additional file 1: Supplementary Figure S1).

\section{Meta-analyses results for polymorphisms in VEGF and $\mathrm{HCC}$}

Nine studies were eligible for the estimation of associations between polymorphisms in VEGF and HCC. VEGF rs699947 (recessive comparison OR $=0.63,95 \% \mathrm{CI}$ 0.41-0.98) and rs3025039 (allele comparison OR $=1.21$, 


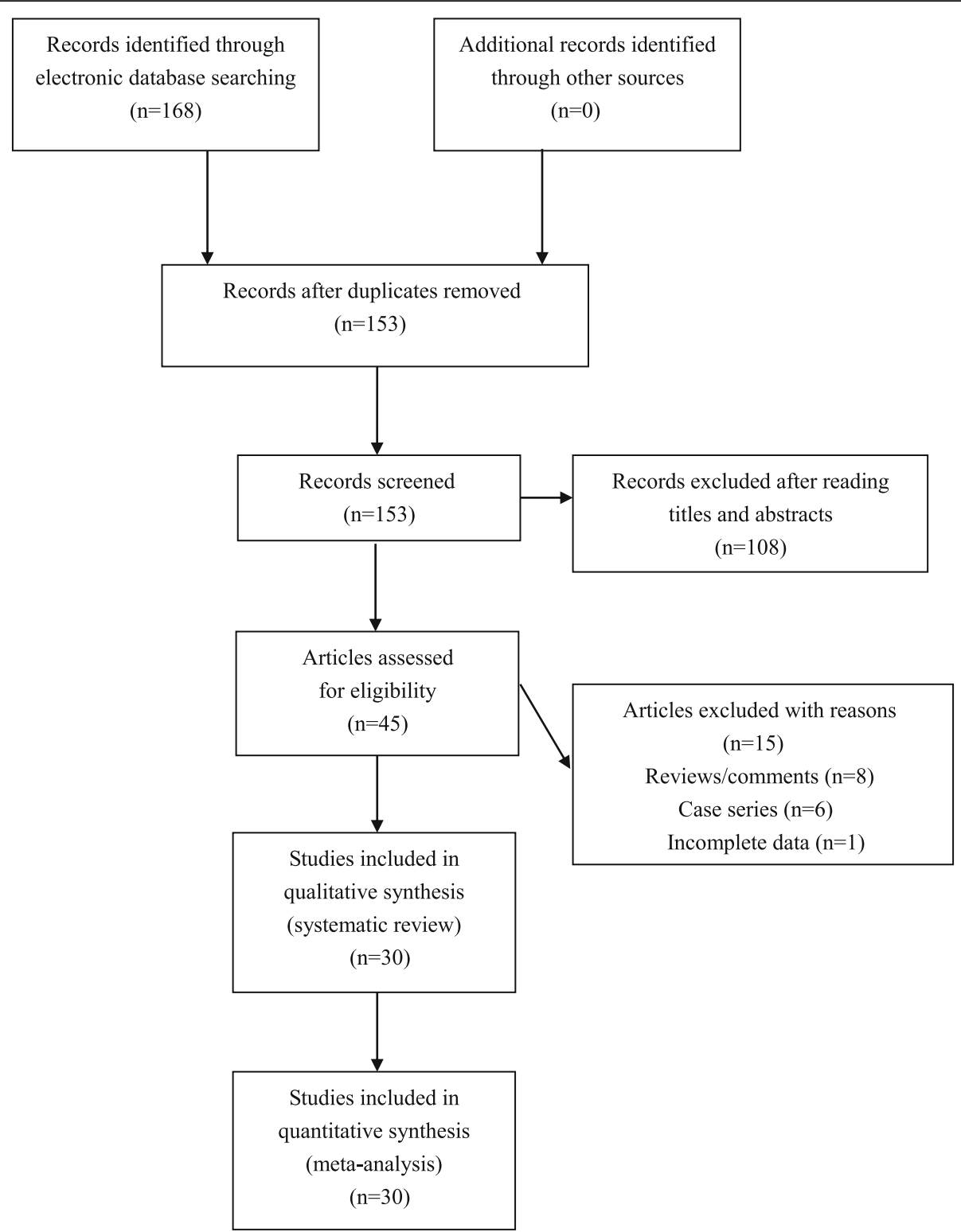

Fig. 1. Flowchart of study selection for the present study

95\% CI 1.00-1.46) polymorphisms were found to be significantly associated with HCC in overall combined analyses. Nevertheless, we did not observe any positive associations in subgroup analyses (see Table 2 and Additional file 1: Supplementary Figure S1).

\section{Meta-analyses results for polymorphisms in IL-18 and HCC}

Ten studies were eligible for the estimation of associations between polymorphisms in $I L-18$ and HCC. $I L-18$ rs1946518 (dominant comparison OR $=0.79,95 \% \mathrm{CI}$ $0.68-0.92$; recessive comparison $\mathrm{OR}=1.26,95 \% \mathrm{CI}$ 1.08-1.48; allele comparison OR $=0.78$, 95\% CI $0.67-$ 0.91) polymorphism was found to be significantly associated with HCC in overall combined analyses. Subgroup analyses showed similar positive findings for rs1946518 polymorphism in East Asians (allele comparison), South Asians (dominant, recessive, and allele comparisons), and those with hepatitis $\mathrm{B}$ virus (HBV) infection (dominant and allele comparisons) (see Table 2 and Additional file 1: Supplementary Figure S1).

Meta-analyses results for polymorphisms in MBL and HCC Five studies were eligible for the estimation of associations between polymorphisms in $M B L$ and $\mathrm{HCC}$. $M B L$ rs7096206 (dominant comparison OR $=0.59,95 \% \mathrm{CI}$ 0.48-0.73; over-dominant comparison OR $=1.59,95 \%$ CI 1.28-1.97; allele comparison: $\mathrm{OR}=0.63,95 \% \mathrm{CI}$ 
Table 1 The characteristics of included studies for this meta-analysis

\begin{tabular}{|c|c|c|c|c|c|c|c|c|c|}
\hline \multirow[t]{2}{*}{ First author, year } & \multirow[t]{2}{*}{ Country } & \multirow[t]{2}{*}{ Ethnicity } & \multirow{2}{*}{$\begin{array}{l}\text { Type } \\
\text { of } \\
\text { disease }\end{array}$} & \multirow[t]{2}{*}{$\begin{array}{l}\text { Medical history } \\
\text { of patients }\end{array}$} & \multirow{2}{*}{$\begin{array}{l}\text { Sample } \\
\text { size } \\
\text { Case/ } \\
\text { control }\end{array}$} & \multicolumn{2}{|c|}{$\begin{array}{l}\text { Genotype distribution } \\
\text { (wtwt/wtmt/mtmt) }\end{array}$} & \multirow{2}{*}{$\begin{array}{l}P \text { value } \\
\text { for } \\
\text { HWE }\end{array}$} & \multirow[t]{2}{*}{$\begin{array}{l}\text { NOS } \\
\text { score }\end{array}$} \\
\hline & & & & & & Cases controls & & & \\
\hline \multicolumn{10}{|l|}{ VDR rs7975232 } \\
\hline Barooah 2019 [11] & India & South Asian & $\mathrm{HCC}$ & NA & $60 / 102$ & $49 / 11 / 0$ & $59 / 35 / 8$ & 0.391 & 8 \\
\hline Falleti 2010 [12] & Italy & Caucasian & $\mathrm{HCC}$ & $\begin{array}{l}\text { Viral } \\
\text { hepatitis 87\% }\end{array}$ & $80 / 160$ & $27 / 38 / 15$ & $53 / 85 / 22$ & 0.189 & 8 \\
\hline Hung 2014 [13] & Taiwan & East Asian & $\mathrm{HCC}$ & NA & $92 / 100$ & $65 / 24 / 3$ & $55 / 40 / 5$ & 0.505 & 8 \\
\hline Yao 2013 [16] & China & East Asian & $\mathrm{HCC}$ & $\begin{array}{l}\text { HBV } 100 \% \text {, } \\
\text { alcohol intake 34.9\% }\end{array}$ & $436 / 532$ & $112 / 216 / 108$ & $114 / 275 / 143$ & 0.395 & 8 \\
\hline \multicolumn{10}{|l|}{ VDR rs1544410 } \\
\hline Barooah 2019 [11] & India & South Asian & $\mathrm{HCC}$ & NA & $60 / 102$ & $52 / 8 / 0$ & $80 / 16 / 6$ & $<0.001$ & 8 \\
\hline Falleti 2010 [12] & Italy & Caucasian & $\mathrm{HCC}$ & $\begin{array}{l}\text { Viral } \\
\text { hepatitis 87\% }\end{array}$ & $80 / 160$ & $33 / 35 / 12$ & $45 / 87 / 28$ & 0.206 & 8 \\
\hline Hung 2014 [13] & Taiwan & East Asian & $\mathrm{HCC}$ & NA & $92 / 100$ & $85 / 7 / 0$ & $89 / 11 / 0$ & 0.560 & 8 \\
\hline Yao 2013 [16] & China & East Asian & $\mathrm{HCC}$ & $\begin{array}{l}\text { HBV } 100 \% \text {, } \\
\text { alcohol intake 34.9\% }\end{array}$ & $436 / 532$ & $112 / 217 / 107$ & $142 / 259 / 131$ & 0.550 & 8 \\
\hline \multicolumn{10}{|l|}{ VDR rs2228570 } \\
\hline Falleti 2010 [12] & Italy & Caucasian & $\mathrm{HCC}$ & Viral hepatitis $87 \%$ & $80 / 160$ & $36 / 36 / 8$ & $69 / 73 / 18$ & 0.843 & 8 \\
\hline Liu 2015 [14] & China & East Asian & $\mathrm{HCC}$ & NA & $105 / 100$ & $41 / 44 / 20$ & $23 / 48 / 29$ & 0.715 & 8 \\
\hline Peng 2014 [15] & China & East Asian & $\mathrm{HCC}$ & $\begin{array}{l}\text { HBV } 100 \% \text {, } \\
\text { alcohol intake } 90.2 \%\end{array}$ & $184 / 296$ & $54 / 90 / 40$ & $77 / 152 / 67$ & 0.628 & 8 \\
\hline Yao 2013 [16] & China & East Asian & $\mathrm{HCC}$ & $\begin{array}{l}\text { HBV } 100 \% \text {, } \\
\text { alcohol intake } 34.9 \%\end{array}$ & $436 / 532$ & 131/198/107 & 102/241/189 & 0.111 & 8 \\
\hline \multicolumn{10}{|l|}{ VDR rs731236 } \\
\hline Barooah 2019 [11] & India & South Asian & $\mathrm{HCC}$ & NA & $60 / 102$ & $48 / 8 / 4$ & $71 / 21 / 10$ & $<0.001$ & 8 \\
\hline Falleti 2010 [12] & Italy & Caucasian & $\mathrm{HCC}$ & $\begin{array}{l}\text { Viral } \\
\text { hepatitis 87\% }\end{array}$ & $80 / 160$ & $32 / 38 / 10$ & $44 / 88 / 28$ & 0.160 & 8 \\
\hline Hung 2014 [13] & Taiwan & East Asian & $\mathrm{HCC}$ & NA & $92 / 100$ & $86 / 6 / 0$ & $86 / 14 / 0$ & 0.452 & 8 \\
\hline Yao 2013 [16] & China & East Asian & $\mathrm{HCC}$ & $\begin{array}{l}\text { HBV } 100 \% \text {, } \\
\text { alcohol intake 34.9\% }\end{array}$ & $436 / 532$ & $115 / 212 / 109$ & $137 / 252 / 143$ & 0.226 & 8 \\
\hline \multicolumn{10}{|l|}{ VEGF rs699947 } \\
\hline Liu 2017 [19] & China & East Asian & $\mathrm{HCC}$ & $\begin{array}{l}\text { HBV } 60.2 \% \text {, } \\
\text { alcohol intake } 60.8 \%\end{array}$ & $476 / 526$ & $301 / 157 / 18$ & $290 / 202 / 34$ & 0.882 & 8 \\
\hline Machado 2014 [20] & Portugal & Caucasian & $\mathrm{HCC}$ & Alcohol intake $100 \%$ & $26 / 101$ & $7 / 14 / 5$ & $19 / 49 / 33$ & 0.914 & 7 \\
\hline Ratnasari 2017 [22] & Indonesia & East Asian & $\mathrm{HCC}$ & HBV58\%, HCV 11\% & $44 / 59$ & $18 / 21 / 5$ & 23/30/6 & 0.402 & 7 \\
\hline Wu 2009 [23] & China & East Asian & $\mathrm{HCC}$ & NA & $92 / 90$ & $48 / 40 / 4$ & $58 / 28 / 4$ & 0.792 & 8 \\
\hline Wu 2013 [24] & China & East Asian & $\mathrm{HCC}$ & HBV48.5\% & $101 / 110$ & 79/21/1 & $91 / 17 / 2$ & 0.271 & 8 \\
\hline \multicolumn{10}{|l|}{ VEGF rs 1570360} \\
\hline Baitello 2016 [17] & Canada & Mixed & $\mathrm{HCC}$ & $\begin{array}{l}\text { HBV 50\%, HCV 21\%, } \\
\text { alcohol intake 56\% }\end{array}$ & $102 / 127$ & $61 / 35 / 6$ & $73 / 47 / 7$ & 0.875 & 8 \\
\hline Wu 2009 [23] & China & East Asian & $\mathrm{HCC}$ & NA & $90 / 99$ & $66 / 24 / 0$ & $72 / 27 / 0$ & 0.116 & 8 \\
\hline Wu 2013 [24] & China & East Asian & $\mathrm{HCC}$ & HBV48.5\% & $101 / 110$ & $83 / 17 / 1$ & $75 / 31 / 4$ & 0.723 & 8 \\
\hline \multicolumn{10}{|l|}{ VEGF rs2010963 } \\
\hline Liu 2017 [19] & China & East Asian & $\mathrm{HCC}$ & $\begin{array}{l}\text { HBV } 60.2 \% \text {, } \\
\text { alcohol intake } 60.8 \%\end{array}$ & $476 / 526$ & $162 / 232 / 82$ & $200 / 248 / 78$ & 0.937 & 8 \\
\hline Ratnasari 2016 [21] & Indonesia & East Asian & $\mathrm{HCC}$ & $\begin{array}{l}\text { HBV } 56.5 \% \\
\text { HCV } 10.8 \%\end{array}$ & $46 / 136$ & 16/29/1 & $26 / 105 / 5$ & $<0.001$ & 7 \\
\hline Wu 2009 [23] & China & East Asian & $\mathrm{HCC}$ & NA & $92 / 99$ & $34 / 40 / 18$ & $34 / 52 / 13$ & 0.320 & 8 \\
\hline
\end{tabular}


Table 1 The characteristics of included studies for this meta-analysis (Continued)

\begin{tabular}{|c|c|c|c|c|c|c|c|c|c|}
\hline \multirow{3}{*}{$\begin{array}{l}\text { First author, year } \\
\text { Wu } 2013 \text { [24] }\end{array}$} & \multirow{3}{*}{$\begin{array}{l}\text { Country } \\
\text { China }\end{array}$} & \multirow{3}{*}{$\begin{array}{l}\text { Ethnicity } \\
\text { East Asian }\end{array}$} & \multirow{3}{*}{$\begin{array}{l}\begin{array}{l}\text { Type } \\
\text { of } \\
\text { disease }\end{array} \\
\text { HCC }\end{array}$} & \multirow{3}{*}{$\begin{array}{l}\text { Medical history } \\
\text { of patients } \\
\text { HBV48.5\% }\end{array}$} & \multirow{3}{*}{$\begin{array}{l}\text { Sample } \\
\text { size } \\
\text { Case/ } \\
\text { control } \\
101 / 110\end{array}$} & \multicolumn{2}{|c|}{$\begin{array}{l}\text { Genotype distribution } \\
\text { (wtwt/wtmt/mtmt) }\end{array}$} & \multirow{3}{*}{$\begin{array}{l}P \text { value } \\
\text { for } \\
\text { HWE } \\
0.506\end{array}$} & \multirow{3}{*}{$\begin{array}{l}\text { NOS } \\
\text { score } \\
8\end{array}$} \\
\hline & & & & & & \multicolumn{2}{|c|}{ Cases controls } & & \\
\hline & & & & & & $28 / 52 / 21$ & $35 / 51 / 24$ & & \\
\hline \multicolumn{10}{|l|}{ VEGF rs3025039 } \\
\hline Baitello 2016 [17] & Canada & Mixed & $\mathrm{HCC}$ & $\begin{array}{l}\text { HBV } 50 \% \text {, HCV 21\%, } \\
\text { alcohol intake 56\% }\end{array}$ & $102 / 127$ & $72 / 30 / 0$ & $90 / 37 / 0$ & 0.055 & 8 \\
\hline Giacalone 2011 [18] & Italy & Caucasian & $\mathrm{HCC}$ & NA & $96 / 162$ & $81 / 14 / 1$ & $120 / 38 / 4$ & 0.636 & 8 \\
\hline Liu 2017 [19] & China & East Asian & $\mathrm{HCC}$ & $\begin{array}{l}\text { HBV } 60.2 \% \\
\text { alcohol intake } 60.8 \%\end{array}$ & $476 / 526$ & $359 / 112 / 5$ & $370 / 140 / 16$ & 0.536 & 8 \\
\hline Wu 2009 [23] & China & East Asian & $\mathrm{HCC}$ & NA & $92 / 99$ & $63 / 26 / 3$ & $68 / 30 / 1$ & 0.239 & 8 \\
\hline Yvamoto 2015 [25] & Brazil & Mixed & $\mathrm{HCC}$ & $\begin{array}{l}\text { Alcohol } \\
\text { intake } 47.1 \%\end{array}$ & $228 / 56$ & $164 / 64 / 0$ & $43 / 13 / 0$ & 0.326 & 7 \\
\hline \multicolumn{10}{|l|}{ IL-18 rs187238 } \\
\hline Bakr 2018 [26] & Egypt & South Asian & $\mathrm{HCC}$ & HCV 100\% & $90 / 90$ & $66 / 22 / 2$ & $33 / 65 / 1$ & $<0.001$ & 8 \\
\hline Bao 2015 [27] & China & East Asian & $\mathrm{HCC}$ & HBV $100 \%$ & $153 / 165$ & $122 / 28 / 3$ & $106 / 54 / 5$ & 0.548 & 8 \\
\hline Chen 2012 [28] & China & East Asian & $\mathrm{HCC}$ & NA & $228 / 300$ & $159 / 59 / 10$ & $173 / 115 / 12$ & 0.183 & 7 \\
\hline Dai 2017 [29] & China & East Asian & $\mathrm{HCC}$ & $\begin{array}{l}\text { HBV } 100 \%, \\
\text { alcohol intake } 42 \%\end{array}$ & $245 / 250$ & $187 / 49 / 9$ & $183 / 65 / 2$ & 0.142 & 8 \\
\hline Karra 2015 [30] & India & South Asian & $\mathrm{HCC}$ & HBV 100\% & $271 / 280$ & $123 / 134 / 14$ & $159 / 108 / 13$ & 0.320 & 7 \\
\hline Kim 2009 [31] & Korea & East Asian & $\mathrm{HCC}$ & HBV 100\% & $56 / 558$ & $37 / 17 / 2$ & $434 / 122 / 2$ & 0.031 & 7 \\
\hline Lau 2016 [32] & Taiwan & East Asian & $\mathrm{HCC}$ & $\begin{array}{l}\text { Alcohol } \\
\text { intake 63.5\% }\end{array}$ & $342 / 559$ & $266 / 73 / 3$ & $476 / 78 / 5$ & 0.370 & 8 \\
\hline Migita 2009 [33] & Japan & East Asian & $\mathrm{HCC}$ & HBV 100\% & $47 / 63$ & $43 / 3 / 1$ & $52 / 10 / 1$ & 0.531 & 7 \\
\hline Teixeira 2009 [34] & Brazil & Mixed & $\mathrm{HCC}$ & $\begin{array}{l}\text { Viral hepatitis } 67.8 \% \text {, } \\
\text { alcohol intake } 63.4 \%\end{array}$ & $112 / 202$ & $57 / 48 / 7$ & $100 / 84 / 18$ & 0.952 & 7 \\
\hline Zhang 2016 [35] & China & East Asian & $\mathrm{HCC}$ & HBV 100\% & $109 / 127$ & $82 / 25 / 2$ & $99 / 24 / 4$ & 0.110 & 8 \\
\hline \multicolumn{10}{|l|}{ IL18 rs1946518 } \\
\hline Bakr 2018 [26] & Egypt & South Asian & $\mathrm{HCC}$ & HCV 100\% & $90 / 99$ & $13 / 34 / 43$ & $17 / 45 / 37$ & 0.603 & 8 \\
\hline Bao 2015 [27] & China & East Asian & $\mathrm{HCC}$ & HBV 100\% & $153 / 165$ & $37 / 73 / 43$ & $41 / 76 / 48$ & 0.322 & 8 \\
\hline Chen 2012 [28] & China & East Asian & $\mathrm{HCC}$ & NA & $228 / 300$ & $47 / 126 / 55$ & $83 / 156 / 61$ & 0.429 & 7 \\
\hline Dai 2017 [29] & China & East Asian & $\mathrm{HCC}$ & $\begin{array}{l}\text { HBV } 100 \% \text {, } \\
\text { alcohol intake } 42 \%\end{array}$ & $247 / 250$ & $62 / 118 / 67$ & $64 / 124 / 62$ & 0.900 & 8 \\
\hline Karra 2015 [30] & India & South Asian & $\mathrm{HCC}$ & HBV 100\% & $271 / 280$ & $70 / 152 / 49$ & $102 / 144 / 34$ & 0.119 & 7 \\
\hline Lau 2016 [32] & Taiwan & East Asian & $\mathrm{HCC}$ & $\begin{array}{l}\text { Alcohol } \\
\text { intake 63.5\% }\end{array}$ & $342 / 559$ & $88 / 167 / 87$ & $148 / 276 / 135$ & 0.777 & 8 \\
\hline Migita 2009 [33] & Japan & East Asian & $\mathrm{HCC}$ & HBV 100\% & $47 / 63$ & $13 / 26 / 8$ & 20/30/13 & 0.777 & 7 \\
\hline Teixeira 2009 [34] & Brazil & Mixed & $\mathrm{HCC}$ & $\begin{array}{l}\text { Viral hepatitis } 67.8 \% \text {, } \\
\text { alcohol intake } 63.4 \%\end{array}$ & $112 / 202$ & $38 / 56 / 18$ & $85 / 105 / 12$ & 0.202 & 7 \\
\hline Zhang 2016 [35] & China & East Asian & $\mathrm{HCC}$ & HBV 100\% & $109 / 127$ & $22 / 55 / 32$ & $38 / 66 / 23$ & 0.127 & 8 \\
\hline \multicolumn{10}{|l|}{ MBL rs7096206 } \\
\hline Eurich 2011 [36] & Germany & Caucasian & $\mathrm{HCC}$ & NA & $62 / 115$ & 27/34/1 & $76 / 37 / 2$ & 0.292 & 7 \\
\hline Gu 2016 [37] & China & East Asian & $\mathrm{HCC}$ & NA & $334 / 171$ & $232 / 95 / 7$ & $131 / 33 / 7$ & 0.015 & 8 \\
\hline Lin 2015 [38] & China & East Asian & $\mathrm{HCC}$ & Alcohol intake $77.7 \%$ & $220 / 220$ & $125 / 86 / 9$ & $153 / 65 / 2$ & 0.082 & 8 \\
\hline Su 2016 [40] & China & East Asian & $\mathrm{HCC}$ & HBV $70.2 \%$ & $315 / 315$ & 207/91/17 & 239/72/4 & 0.583 & 8 \\
\hline MBL rs 1800450 & & & & NA & & & & & \\
\hline Gu 2016 [37] & China & East Asian & $\mathrm{HCC}$ & NA & $334 / 171$ & 234/89/11 & $104 / 59 / 8$ & 0.920 & 8 \\
\hline Segat 2008 [39] & Italy & Caucasian & $\mathrm{HCC}$ & NA & $215 / 164$ & $127 / 78 / 10$ & $102 / 49 / 13$ & 0.050 & 7 \\
\hline
\end{tabular}


Table 1 The characteristics of included studies for this meta-analysis (Continued)

\begin{tabular}{|c|c|c|c|c|c|c|c|c|c|}
\hline \multirow[t]{2}{*}{ First author, year } & \multirow[t]{2}{*}{ Country } & \multirow[t]{2}{*}{ Ethnicity } & \multirow{2}{*}{$\begin{array}{l}\text { Type } \\
\text { of } \\
\text { disease }\end{array}$} & \multirow[t]{2}{*}{$\begin{array}{l}\text { Medical history } \\
\text { of patients }\end{array}$} & \multirow{2}{*}{$\begin{array}{l}\text { Sample } \\
\text { size } \\
\text { Case/ } \\
\text { control }\end{array}$} & \multicolumn{2}{|c|}{$\begin{array}{l}\text { Genotype distribution } \\
\text { (wtwt/wtmt/mtmt) }\end{array}$} & \multirow{2}{*}{$\begin{array}{l}P \text { value } \\
\text { for } \\
\text { HWE }\end{array}$} & \multirow[t]{2}{*}{$\begin{array}{l}\text { NOS } \\
\text { score }\end{array}$} \\
\hline & & & & & & Cases cont & & & \\
\hline Su 2016 [40] & China & East Asian & $\mathrm{HCC}$ & HBV $70.2 \%$ & $308 / 315$ & $208 / 88 / 20$ & $239 / 69 / 7$ & 0.450 & 8 \\
\hline
\end{tabular}

Abbreviations: HWE Hardy-Weinberg equilibrium, NOS Newcastle-Ottawa scale, NA not available, HBV hepatitis B virus infection, $H C V$ hepatitis $C$ virus infection

00.53-0.76) polymorphism was found to be significantly associated with HCC in overall combined analyses. Subgroup analyses showed similar positive findings for rs7096206 polymorphism in East Asians (dominant, over-dominant, and allele comparisons) (see Table 2 and Additional file 1: Supplementary Figure S1).

\section{Sensitivity analyses}

We examined the stability of combined results by deleting one study each time and combining the results of the remaining studies. The trends of associations remained consistent in sensitivity analyses, which indicated that the combined results were statistically stable.

\section{Publication biases}

Funnels plots were employed to estimate whether our combined results may be influenced by publication biases. Funnel plots of every comparison were symmetrical, which indicated that the combined results were unlikely to be seriously impacted by overt publication biases.

\section{Discussion}

The combined results of this meta-analysis revealed that VDR rs7975232, VDR rs2228570, VEGF rs699947, VEGF rs3025039, $I L-18$ rs1946518, and $M B L$ rs7096206 polymorphisms were significantly associated with susceptibility to $\mathrm{HCC}$ in certain populations. The trends of associations remained consistent in sensitivity analyses, which indicated that the combined results were statistically stable.

To better understand the combined results of this meta-analysis, some points should be considered. First, past basic studies revealed that all investigated polymorphisms were either correlated with altered transcription activity or protein structure [45-48]. So, these variations may influence the biological function of $V D R / V E G F / I L-$ $18 / M B L$, result in immune dysfunction, cause chronic inflammatory hepatocellular injury, and ultimately confer susceptibility to HCC. Thus, our meta-analysis may be statistically insufficient to observe the real underlying associations between polymorphisms in VDR/VEGF/IL$18 / M B L$ and HCC in certain subgroups. Therefore, future studies still need to confirm our findings. Second, we noticed that most eligible studies were from Asian countries, whereas studies in other countries were highly scarce, so scholars from European and African countries should also try to examine associations between polymorphisms in VDR/VEGF/IL-18/MBL and HCC. Besides, considering the functional importance of VDR/ VEGF/IL-18/MBL in regulating inflammatory reactions and angiogenesis, future studies also need to test the relationship between polymorphisms in VDR/VEGF/IL-18/ $M B L$ and other types of malignancies. Third, the etiology of HCC is very complicated, so we highly recommend further genetic association studies to explore the effects of haplotypes and gene-gene interactions on disease susceptibility [49]. Fourth, we aimed to investigate associations between all polymorphisms in VDR/VEGF/ $I L-18 / M B L$ and HCC in the very beginning. However, we did not find any study on other VDR/VEGF/IL-18/ $M B L$ polymorphisms, so we only focused on 12 polymorphisms in this meta-analysis. Fifth, it is worth noting that Zhu et al. [50] also performed a meta-analysis about $I L-18$ polymorphisms and HCC in 2016. Based on combined analyses of eight eligible studies with 3572 subjects, they did not find any positive results regarding $I L$ 18 polymorphisms and HCC in general or subgroup analyses. Since our pooled analyses about $I L-18$ polymorphisms were based on more eligible studies and larger sample sizes, our results should be more statistically robust. Nevertheless, studies with larger sample sizes are still warranted to test the genetic associations between $I L-18$ polymorphisms and $\mathrm{HCC}$ in the future.

Some limitations of this meta-analysis should also be mentioned. Firstly, the results regarding associations between polymorphisms in VDR/VEGF/IL-18/MBL and $\mathrm{HCC}$ were based on combining unadjusted findings of eligible studies due to the lack of raw data [51]. Secondly, the relationship between polymorphisms in $V D R / V E G F / I L-18 / M B L$ and HCC may also be affected by environmental factors. Unfortunately, the majority of eligible studies only focused on associations between polymorphisms in VDR/VEGF/IL-18/MBL and HCC, so we could not explore genetic-environmental interactions in this meta-analysis [52]. Thirdly, grey literatures were not searched. So although funnel plots of every comparison were symmetrical, it is still possible that the combined results may be affected by publication biases [53]. 
Table 2 Meta-analyses results of the current study

\begin{tabular}{|c|c|c|c|c|c|c|c|c|c|}
\hline \multirow[t]{2}{*}{ Variables } & \multirow{2}{*}{$\begin{array}{l}\text { Sample } \\
\text { size }\end{array}$} & \multicolumn{2}{|l|}{ Dominant comparison } & \multicolumn{2}{|c|}{ Recessive comparison } & \multicolumn{2}{|c|}{ Over-dominant comparison } & \multicolumn{2}{|c|}{ Allele comparison } \\
\hline & & \multicolumn{2}{|l|}{$\begin{array}{l}P \text { value OR }(95 \% \mathrm{Cl}) P^{2} \\
\text { statistic }\end{array}$} & \multirow[t]{2}{*}{$\begin{array}{l}P \\
\text { value }\end{array}$} & \multirow[t]{2}{*}{$\begin{array}{l}\text { OR }(95 \% \mathrm{Cl}) P^{2} \\
\text { statistic }\end{array}$} & \multicolumn{2}{|l|}{$\begin{array}{l}P \text { value OR }(95 \% \mathrm{Cl}) \\
P^{2} \text { statistic }\end{array}$} & \multirow[t]{2}{*}{$P$ value } & \multirow[t]{2}{*}{$\begin{array}{l}\text { OR }(95 \% \text { Cl } \\
P^{2} \text { statistic }\end{array}$} \\
\hline VDR rs797 & & & & & & & & & \\
\hline Overall & $668 / 894$ & $\begin{array}{l}0.03 \\
60 \%\end{array}$ & $\begin{array}{l}1.58 \\
(1.04-2.39)\end{array}$ & $\begin{array}{l}0.42 \\
31 \%\end{array}$ & $0.90(0.69-1.17)$ & $\begin{array}{l}0.03 \\
44 \%\end{array}$ & $\begin{array}{l}0.80 \\
(0.65-0.98)\end{array}$ & $\begin{array}{l}0.09 \\
76 \%\end{array}$ & $\begin{array}{l}1.41 \\
(0.94-2.12)\end{array}$ \\
\hline $\begin{array}{l}\text { East } \\
\text { Asian }\end{array}$ & $528 / 632$ & $\begin{array}{l}0.02 \\
40 \%\end{array}$ & $\begin{array}{l}1.39 \\
(1.06-1.81)\end{array}$ & $\begin{array}{l}0.40 \\
0 \%\end{array}$ & $0.88(0.67-1.17)$ & $\begin{array}{l}0.28 \\
62 \%\end{array}$ & $\begin{array}{l}0.75 \\
(0.45-1.26)\end{array}$ & $\begin{array}{l}0.17 \\
55 \%\end{array}$ & $\begin{array}{l}1.30 \\
(0.89-1.89)\end{array}$ \\
\hline \multicolumn{10}{|c|}{ VDR rs 1544410} \\
\hline Overall & $668 / 894$ & $\begin{array}{l}0.26 \\
44 \%\end{array}$ & $\begin{array}{l}1.15 \\
(0.90-1.45)\end{array}$ & $\begin{array}{l}0.62 \\
8 \%\end{array}$ & $0.93(0.71-1.22)$ & $\begin{array}{l}0.54 \\
0 \%\end{array}$ & $\begin{array}{l}0.93 \\
(0.75-1.16)\end{array}$ & $\begin{array}{l}0.30 \\
50 \%\end{array}$ & $\begin{array}{l}1.09 \\
(0.93-1.27)\end{array}$ \\
\hline $\begin{array}{l}\text { East } \\
\text { Asian }\end{array}$ & $528 / 632$ & $\begin{array}{l}0.98 \\
0 \%\end{array}$ & $\begin{array}{l}1.00 \\
(0.74-1.34)\end{array}$ & $\begin{array}{l}0.91 \\
0 \%\end{array}$ & $0.98(0.75-1.30)$ & $\begin{array}{l}0.90 \\
0 \%\end{array}$ & $\begin{array}{l}1.02 \\
(0.79-1.30)\end{array}$ & $\begin{array}{l}0.96 \\
0 \%\end{array}$ & $\begin{array}{l}1.00 \\
(0.83-1.19)\end{array}$ \\
\hline \multicolumn{10}{|c|}{ VDR rs 2228570} \\
\hline Overall & $\begin{array}{l}805 / \\
1088\end{array}$ & $\begin{array}{l}<0.0001 \\
46 \%\end{array}$ & $\begin{array}{l}1.54 \\
(1.25-1.89)\end{array}$ & $\begin{array}{l}0.0004 \\
19 \%\end{array}$ & $0.67(0.54-0.84)$ & $\begin{array}{l}0.58 \\
0 \%\end{array}$ & $\begin{array}{l}0.95 \\
(0.79-1.14)\end{array}$ & $\begin{array}{l}0.01 \\
59 \%\end{array}$ & $\begin{array}{l}1.34 \\
(1.06-1.68)\end{array}$ \\
\hline $\begin{array}{l}\text { East } \\
\text { Asian }\end{array}$ & $725 / 928$ & $\begin{array}{l}<0.0001 \\
45 \%\end{array}$ & $\begin{array}{l}1.63 \\
(1.31-2.04)\end{array}$ & $\begin{array}{l}0.0003 \\
40 \%\end{array}$ & $0.66(0.53-0.83)$ & $\begin{array}{l}0.58 \\
0 \%\end{array}$ & $\begin{array}{l}0.95 \\
(0.78-1.15)\end{array}$ & $\begin{array}{l}0.01 \\
65 \%\end{array}$ & $\begin{array}{l}1.40 \\
(1.08-1.82)\end{array}$ \\
\hline \multicolumn{10}{|c|}{ VDR rs731236 } \\
\hline Overall & $668 / 894$ & $\begin{array}{l}0.06 \\
43 \%\end{array}$ & $\begin{array}{l}1.25 \\
(0.99-1.58)\end{array}$ & $\begin{array}{l}0.26 \\
0 \%\end{array}$ & $0.86(0.66-1.12)$ & $\begin{array}{l}0.42 \\
38 \%\end{array}$ & $\begin{array}{l}0.92 \\
(0.74-1.14)\end{array}$ & $\begin{array}{l}0.06 \\
42 \%\end{array}$ & $\begin{array}{l}1.16 \\
(0.99-1.36)\end{array}$ \\
\hline $\begin{array}{l}\text { East } \\
\text { Asian }\end{array}$ & $528 / 632$ & $\begin{array}{l}0.44 \\
57 \%\end{array}$ & $\begin{array}{l}1.34 \\
(0.64-2.82)\end{array}$ & $\begin{array}{l}0.51 \\
0 \%\end{array}$ & $0.91(0.68-1.21)$ & $\begin{array}{l}0.54 \\
66 \%\end{array}$ & $\begin{array}{l}0.77 \\
(0.33-1.78)\end{array}$ & $\begin{array}{l}0.39 \\
55 \%\end{array}$ & $\begin{array}{l}1.08 \\
(0.91-1.29)\end{array}$ \\
\hline \multicolumn{10}{|c|}{ VEGF rs699947 } \\
\hline Overall & $739 / 886$ & $\begin{array}{l}0.92 \\
54 \%\end{array}$ & $\begin{array}{l}1.02 \\
(0.69-1.52)\end{array}$ & $\begin{array}{l}0.04 \\
0 \%\end{array}$ & $0.63(0.41-0.98)$ & $\begin{array}{l}0.61 \\
45 \%\end{array}$ & $\begin{array}{l}0.95 \\
(0.77-1.17)\end{array}$ & $\begin{array}{l}0.61 \\
51 \%\end{array}$ & $\begin{array}{l}1.08 \\
(0.80-1.46)\end{array}$ \\
\hline $\begin{array}{l}\text { East } \\
\text { Asian }\end{array}$ & 713/785 & $\begin{array}{l}0.84 \\
64 \%\end{array}$ & $\begin{array}{l}1.05 \\
(0.66-1.66)\end{array}$ & $\begin{array}{l}0.10 \\
0 \%\end{array}$ & $0.67(0.41-1.08)$ & $\begin{array}{l}0.70 \\
56 \%\end{array}$ & $\begin{array}{l}1.08 \\
(0.72-1.65)\end{array}$ & $\begin{array}{l}0.99 \\
59 \%\end{array}$ & $\begin{array}{l}1.00 \\
(0.70-1.42)\end{array}$ \\
\hline \multicolumn{10}{|c|}{ VEGF rs 1570360} \\
\hline Overall & $293 / 336$ & $\begin{array}{l}0.12 \\
37 \%\end{array}$ & $\begin{array}{l}1.31 \\
(0.93-1.85)\end{array}$ & $\begin{array}{l}0.57 \\
19 \%\end{array}$ & $0.75(0.29-1.98)$ & $\begin{array}{l}0.17 \\
7 \%\end{array}$ & $\begin{array}{l}0.78 \\
(0.55-1.11)\end{array}$ & $\begin{array}{l}0.13 \\
49 \%\end{array}$ & $\begin{array}{l}1.26 \\
(0.94-1.70)\end{array}$ \\
\hline $\begin{array}{l}\text { East } \\
\text { Asian }\end{array}$ & 191/209 & $\begin{array}{l}0.28 \\
60 \%\end{array}$ & $\begin{array}{l}1.49 \\
(0.72-3.06)\end{array}$ & $\begin{array}{l}0.24 \\
0 \%\end{array}$ & $0.27(0.03-2.41)$ & $\begin{array}{l}0.15 \\
44 \%\end{array}$ & $\begin{array}{l}0.71 \\
(0.45-1.13)\end{array}$ & $\begin{array}{l}0.28 \\
64 \%\end{array}$ & $\begin{array}{l}1.46 \\
(0.73-2.91)\end{array}$ \\
\hline \multicolumn{10}{|c|}{ VEGF rs2010963 } \\
\hline Overall & $715 / 871$ & $\begin{array}{l}0.79 \\
55 \%\end{array}$ & $\begin{array}{l}1.05 \\
(0.72-1.54)\end{array}$ & $\begin{array}{l}0.26 \\
0 \%\end{array}$ & $1.17(0.89-1.55)$ & $\begin{array}{l}0.80 \\
48 \%\end{array}$ & $\begin{array}{l}0.97 \\
(0.80-1.19)\end{array}$ & $\begin{array}{l}0.32 \\
13 \%\end{array}$ & $\begin{array}{l}0.93 \\
(0.81-1.07)\end{array}$ \\
\hline $\begin{array}{l}\text { East } \\
\text { Asian }\end{array}$ & $715 / 871$ & $\begin{array}{l}0.79 \\
55 \%\end{array}$ & $\begin{array}{l}1.05 \\
(0.72-1.54)\end{array}$ & $\begin{array}{l}0.26 \\
0 \%\end{array}$ & $1.17(0.89-1.55)$ & $\begin{array}{l}0.80 \\
48 \%\end{array}$ & $\begin{array}{l}0.97 \\
(0.80-1.19)\end{array}$ & $\begin{array}{l}0.32 \\
13 \%\end{array}$ & $\begin{array}{l}0.93 \\
(0.81-1.07)\end{array}$ \\
\hline \multicolumn{10}{|c|}{ VEGF rs3025039 } \\
\hline Overall & $994 / 970$ & $\begin{array}{l}0.08 \\
12 \%\end{array}$ & $\begin{array}{l}1.20 \\
(0.98-1.48)\end{array}$ & $\begin{array}{l}0.08 \\
38 \%\end{array}$ & $0.50(0.23-1.09)$ & $\begin{array}{l}0.21 \\
0 \%\end{array}$ & $\begin{array}{l}0.87 \\
(0.71-1.08)\end{array}$ & $\begin{array}{l}0.05 \\
28 \%\end{array}$ & $\begin{array}{l}1.21 \\
(1.00-1.46)\end{array}$ \\
\hline $\begin{array}{l}\text { East } \\
\text { Asian }\end{array}$ & $568 / 625$ & $\begin{array}{l}0.10 \\
0 \%\end{array}$ & $\begin{array}{l}1.24 \\
(0.96-1.59)\end{array}$ & $\begin{array}{l}0.87 \\
69 \%\end{array}$ & $0.83(0.09-7.41)$ & $\begin{array}{l}0.25 \\
0 \%\end{array}$ & $\begin{array}{l}0.86 \\
(0.66-1.11)\end{array}$ & $\begin{array}{l}0.06 \\
34 \%\end{array}$ & $\begin{array}{l}1.24 \\
(0.99-1.56)\end{array}$ \\
\hline \multicolumn{10}{|c|}{ IL-18 rs187238 } \\
\hline Overall & $\begin{array}{l}1653 / \\
2594\end{array}$ & $\begin{array}{l}0.38 \\
85 \%\end{array}$ & $\begin{array}{l}1.19 \\
(0.81-1.77)\end{array}$ & $\begin{array}{l}0.50 \\
16 \%\end{array}$ & $1.14(0.78-1.66)$ & $\begin{array}{l}0.26 \\
88 \%\end{array}$ & $\begin{array}{l}0.77 \\
(0.49-1.21)\end{array}$ & $\begin{array}{l}0.56 \\
78 \%\end{array}$ & $\begin{array}{l}1.09 \\
(0.82-1.43)\end{array}$ \\
\hline $\begin{array}{l}\text { East } \\
\text { Asian }\end{array}$ & $\begin{array}{l}1180 / \\
2022\end{array}$ & $\begin{array}{l}0.62 \\
81 \%\end{array}$ & $\begin{array}{l}1.11 \\
(0.73-1.70)\end{array}$ & $\begin{array}{l}0.27 \\
33 \%\end{array}$ & $1.33(0.80-2.22)$ & $\begin{array}{l}0.49 \\
81 \%\end{array}$ & $\begin{array}{l}0.86 \\
(0.55-1.34)\end{array}$ & $\begin{array}{l}0.76 \\
78 \%\end{array}$ & $\begin{array}{l}1.06 \\
(0.74-1.50)\end{array}$ \\
\hline $\begin{array}{l}\text { South } \\
\text { Asian }\end{array}$ & $361 / 370$ & $\begin{array}{l}0.60 \\
97 \%\end{array}$ & $\begin{array}{l}1.70 \\
(0.24-12.29)\end{array}$ & $\begin{array}{l}0.65 \\
0 \%\end{array}$ & $1.19(0.57-2.47)$ & $\begin{array}{l}0.53 \\
98 \%\end{array}$ & $\begin{array}{l}0.45 \\
(0.04-5.35)\end{array}$ & $\begin{array}{l}0.69 \\
92 \%\end{array}$ & $\begin{array}{l}1.25 \\
(0.42-3.66)\end{array}$ \\
\hline HBV & $81 / 1443$ & $\begin{array}{l}0.90 \\
78 \%\end{array}$ & $\begin{array}{l}1.03 \\
(0.65-1.63)\end{array}$ & $\begin{array}{l}0.23 \\
43 \%\end{array}$ & $1.38(0.81-2.33)$ & $\begin{array}{l}0.73 \\
78 \%\end{array}$ & $\begin{array}{l}0.92 \\
(0.57-1.48)\end{array}$ & $\begin{array}{l}0.96 \\
74 \%\end{array}$ & $\begin{array}{l}1.01 \\
(0.70-1.46)\end{array}$ \\
\hline
\end{tabular}


Table 2 Meta-analyses results of the current study (Continued)

\begin{tabular}{|c|c|c|c|c|c|c|c|c|c|}
\hline \multirow[t]{2}{*}{ Variables } & \multirow{2}{*}{$\begin{array}{l}\text { Sample } \\
\text { size }\end{array}$} & \multirow{2}{*}{\multicolumn{2}{|c|}{$\begin{array}{l}\text { Dominant comparison } \\
P \text { value OR }(95 \% C l) I^{2} \\
\text { statistic }\end{array}$}} & \multicolumn{2}{|c|}{ Recessive comparison } & \multicolumn{2}{|c|}{ Over-dominant comparison } & \multicolumn{2}{|c|}{ Allele comparison } \\
\hline & & & & $\begin{array}{l}P \\
\text { value }\end{array}$ & $\begin{array}{l}\text { OR }(95 \% \mathrm{Cl}) P^{2} \\
\text { statistic }\end{array}$ & $\begin{array}{l}\text { value OR }(95 \% \mathrm{Cl}) \\
P^{2} \text { statistic }\end{array}$ & & $P$ value & $\begin{array}{l}\text { OR }(95 \% \mathrm{Cl}) \\
\text { P }^{2} \text { statistic }\end{array}$ \\
\hline Overall & $\begin{array}{l}1599 / \\
2045\end{array}$ & $\begin{array}{l}0.002 \\
0 \%\end{array}$ & $\begin{array}{l}0.79 \\
(0.68-0.92)\end{array}$ & $\begin{array}{l}0.004 \\
30 \%\end{array}$ & $1.26(1.08-1.48)$ & $\begin{array}{l}0.75 \\
0 \%\end{array}$ & $\begin{array}{l}1.02 \\
(0.90-1.17)\end{array}$ & $\begin{array}{l}0.002 \\
59 \%\end{array}$ & $\begin{array}{l}0.78 \\
(0.67-0.91)\end{array}$ \\
\hline $\begin{array}{l}\text { East } \\
\text { Asian }\end{array}$ & $\begin{array}{l}1126 / \\
1464\end{array}$ & $\begin{array}{l}0.09 \\
0 \%\end{array}$ & $\begin{array}{l}0.86 \\
(0.71-1.02)\end{array}$ & $\begin{array}{l}0.15 \\
0 \%\end{array}$ & $1.14(0.95-1.37)$ & $\begin{array}{l}0.79 \\
0 \%\end{array}$ & $\begin{array}{l}1.02 \\
(0.87-1.19)\end{array}$ & $\begin{array}{l}0.04 \\
68 \%\end{array}$ & $\begin{array}{l}0.80 \\
(0.65-0.99)\end{array}$ \\
\hline $\begin{array}{l}\text { South } \\
\text { Asian }\end{array}$ & $589 / 679$ & $\begin{array}{l}0.001 \\
0 \%\end{array}$ & $\begin{array}{l}0.66 \\
(0.51-0.85)\end{array}$ & $\begin{array}{l}0.02 \\
0 \%\end{array}$ & $1.57(1.09-2.27)$ & $\begin{array}{l}0.98 \\
54 \%\end{array}$ & $\begin{array}{l}0.99 \\
(0.61-1.61)\end{array}$ & $\begin{array}{l}0.002 \\
0 \%\end{array}$ & $\begin{array}{l}0.72 \\
(0.59-0.89)\end{array}$ \\
\hline HBV & $827 / 885$ & $\begin{array}{l}0.01 \\
9 \%\end{array}$ & $\begin{array}{l}0.77 \\
(0.62-0.95\end{array}$ & $\begin{array}{l}0.06 \\
21 \%\end{array}$ & $1.25(0.99-1.57)$ & $\begin{array}{l}0.52 \\
0 \%\end{array}$ & $\begin{array}{l}1.06 \\
(0.88-1.29)\end{array}$ & $\begin{array}{l}0.03 \\
73 \%\end{array}$ & $\begin{array}{l}0.73 \\
(0.55-0.96)\end{array}$ \\
\hline \multicolumn{10}{|c|}{ MBL rs7096206 } \\
\hline Overall & $931 / 821$ & $\begin{array}{l}<0.00010 .59(0.48-0.73) \\
0 \%\end{array}$ & & $\begin{array}{l}0.37 \\
70 \%\end{array}$ & $1.81(0.50-6.59)$ & $\begin{array}{l}<0.0001 \\
0 \%\end{array}$ & $\begin{array}{l}1.59 \\
(1.28-1.97)\end{array}$ & $\begin{array}{l}<0.0001 \\
0 \%\end{array}$ & $\begin{array}{l}0.63 \\
(0.53-0.76)\end{array}$ \\
\hline $\begin{array}{l}\text { East } \\
\text { Asian }\end{array}$ & $869 / 706$ & $\begin{array}{l}<0.0001 \\
0 \%\end{array}$ & $\begin{array}{l}0.62 \\
(0.50-0.78)\end{array}$ & $\begin{array}{l}0.35 \\
79 \%\end{array}$ & $2.08(0.44-9.80)$ & $\begin{array}{l}0.0005 \\
0 \%\end{array}$ & $\begin{array}{l}1.50 \\
(1.19-1.88)\end{array}$ & $\begin{array}{l}<0.0001 \\
4 \%\end{array}$ & $\begin{array}{l}0.65 \\
(0.53-0.79)\end{array}$ \\
\hline \multicolumn{10}{|c|}{ MBL rs1800450 } \\
\hline Overall & $857 / 650$ & $\begin{array}{l}0.85 \\
79 \%\end{array}$ & $\begin{array}{l}0.95 \\
(0.58-1.55)\end{array}$ & $\begin{array}{l}0.91 \\
77 \%\end{array}$ & $1.06(0.37-3.06)$ & $\begin{array}{l}0.70 \\
75 \%\end{array}$ & $\begin{array}{l}1.10 \\
(0.69-1.74)\end{array}$ & $\begin{array}{l}0.95 \\
80 \%\end{array}$ & $\begin{array}{l}0.99 \\
(0.65-1.50)\end{array}$ \\
\hline $\begin{array}{l}\text { East } \\
\text { Asian }\end{array}$ & $642 / 486$ & $\begin{array}{l}0.99 \\
90 \%\end{array}$ & $\begin{array}{l}0.99 \\
(0.44-2.23)\end{array}$ & $\begin{array}{l}0.61 \\
81 \%\end{array}$ & $1.47(0.34-6.30)$ & $\begin{array}{l}0.99 \\
86 \%\end{array}$ & $\begin{array}{l}1.00 \\
(0.49-2.03)\end{array}$ & $\begin{array}{l}0.95 \\
90 \%\end{array}$ & $\begin{array}{l}0.98 \\
(0.48-1.99)\end{array}$ \\
\hline
\end{tabular}

Abbreviations: $O R$ odds ratio, $\mathrm{Cl}$ confidence interval, $N A$ not available, $H B V$ hepatitis $\mathrm{B}$ virus infection

The values in italics represent that there is statistically significant differences between cases and controls

\section{Conclusion}

In summary, this meta-analysis proved that $V D R$ rs7975232, VDR rs2228570, VEGF rs699947, VEGF rs3025039, $I L-18$ rs1946518, and $M B L$ rs7096206 polymorphisms may confer susceptibility to HCC in certain populations. These results also indicated that VDR, VEGF, IL-18, and MBL may involve in the development of HCC. However, the combined results of this metaanalysis should still be verified by studies with larger sample sizes.

\section{Supplementary information}

Supplementary information accompanies this paper at https://doi.org/10. 1186/s12957-019-1748-8.

Additional file 1: Figure S1. Forest plots of investigated polymorphisms.

\section{Abbreviations}

VDR: Vitamin D receptor; VEGF: Vascular endothelial growth factor; MBL: Mannose-binding lectin; IL-18: Interleukin-18; HCC: Hepatocellular carcinoma; HWE: Hardy-Weinberg equilibrium; NOS: Newcastle-Ottawa scale; OR: Odds ratios; Cl: Confidence intervals

\section{Acknowledgements}

None.

\section{Ethical approval and consent to participate}

Not applicable.

\section{Informed consent}

For this type of study formal consent is not required.

\section{Authors' contributions}

YQ and YH conceived and designed the study. YQ and JY conducted the literature review. TQ analyzed the data. YQ and YH drafted the manuscript. All authors have read and approved the final manuscript.

\section{Funding}

This article was funded by the Research Project of Guangxi Autonomous Region Ministry of Education (2019KY0536). Fund Project: Department of education of Guangxi Autonomous Region project, No.: $2019 k y 0536$.

Availability of data and materials

The current study was based on the results of relevant published studies.

Consent for publication

Not applicable.

Competing interests

The authors declare that they have no competing interests.

\section{Author details}

${ }^{1}$ Department of Clinical Laboratory, Affiliated Hospital of Guilin Medical University, Guilin, Guangxi, China. ${ }^{2}$ Department of Radiology, Affiliated Hospital of Guilin Medical University, No. 15 of Lequn Road, Guilin 540001, Guangxi, China.

Received: 16 August 2019 Accepted: 11 November 2019

Published online: 12 December 2019

References

1. Siegel RL, Miller KD, Jemal A. Cancer statistics, 2017. CA Cancer J Clin. 2017; 67:7-30.

2. Marcellin P, Kutala BK. Liver diseases: A major, neglected global public health problem requiring urgent actions and large-scale screening. Liver Int. 2018;38(Suppl 1):2-6.

3. Asrani SK, Devarbhavi H, Eaton J, Kamath PS. Burden of liver diseases in the world. J Hepatol. 2019;70:151-71.

4. Ghouri YA, Mian I, Rowe JH. Review of hepatocellular carcinoma: Epidemiology, etiology, and carcinogenesis. J Carcinog. 2017;16:1. 
5. Zucman-Rossi J, Villanueva A, Nault JC, Llovet JM. Genetic landscape and biomarkers of hepatocellular carcinoma. Gastroenterology. 2015;149:1226-39.

6. Niu ZS, Niu XJ, Wang WH. Genetic alterations in hepatocellular carcinoma: An update. World J Gastroenterol. 2016;22:9069-95.

7. Swierzko AS, Kilpatrick DC, Cedzynski M. Mannan-binding lectin in malignancy. Mol Immunol. 2013;55:16-21.

8. Esmailbeig M, Ghaderi A. Interleukin-18: a regulator of cancer and autoimmune diseases. Eur Cytokine Netw. 2017;28:127-40.

9. Campbell MJ, Trump DL. Vitamin D Receptor Signaling and Cancer. Endocrinol Metab Clin North Am. 2017:46:1009-38.

10. Carmeliet P. VEGF as a key mediator of angiogenesis in cancer. Oncology. 2005;69(Suppl 3):4-10.

11. Barooah P, Saikia S, Bharadwaj R, Sarmah P, Bhattacharyya M, Goswami B, Medhi S. Role of VDR, GC, and CYP2R1 Polymorphisms in the development of hepatocellular carcinoma in hepatitis C virus-infected patients. Genet Test Mol Biomarkers. 2019;23:325-31.

12. Falleti $E$, Bitetto $D$, Fabris $C$, Cussigh $A$, Fontanini $E$, Fornasiere $E$, Fumolo $E$, Bignulin S, Cmet S, Minisini R, Pirisi M, Toniutto P. Vitamin D receptor gene polymorphisms and hepatocellular carcinoma in alcoholic cirrhosis. World J Gastroenterol. 2010;16:3016-24.

13. Hung $\mathrm{CH}$, Chiu YC, Hu TH, Chen CH, Lu SN, Huang CM, Wang JH, Lee CM. Significance of vitamin d receptor gene polymorphisms for risk the carcinoma in chronic hepatitis C. Transl Oncol. 2014;7:503-7.

14. Liu Q, Pan G, Zhen M. Study on correlation between Fok I polymorphism of VDR gene and susceptibility to hepatocellular carcinoma. J Chengdu Med College. 2015; 28: 369-371. [Article in Chinese]

15. Peng Q, Yang S, Lao X, Li R, Chen Z, Wang J, Qin X, Li S. Association of single nucleotide polymorphisms in VDR and DBP genes with HBV-related hepatocellular carcinoma risk in a Chinese population. PLoS One. 2014;9: e116026.

16. Yao X, Zeng H, Zhang G, Zhou W, Yan Q, Dai L, Wang X. The associated ion between the VDR gene polymorphisms and susceptibility to hepatocellular carcinoma and the clinicopathological features in subjects infected with HBV. Biomed Res Int. 2013;2013:953974.

17. Baitello ME, Tenani GD, Ferreira RF, Nogueira V, Pinhel MA, da Silva RC, da Silva RF, Fucuta PD, de Godoy MF, Souza DR. VEGF polymorphisms related to higher serum levels of protein identify patients with hepatocellular carcinoma. Can J Gastroenterol Hepatol. 2016:2016:9607054.

18. Giacalone A, Montalto G, Giannitrapani L, Balasus D, Terranova A, Cervello $M$, Soresi $M$, Marasà L. Association between single nucleotide polymorphisms in the cyclooxygenase-2, tumor necrosis factor- $a$, and vascular endothelial growth factor-A genes, and susceptibility to hepatocellular carcinoma. OMICS. 2011;15:193-6.

19. Liu F, Luo L, Wei Y, Wang W, Wen T, Yang J, Xu M, Li B. Association of VEGFA polymorphisms with susceptibility and clinical outcome of hepatocellular carcinoma in a Chinese Han population. Oncotarget. 2017;8: 16488-97.

20. Machado MV, Janeiro A, Miltenberger-Miltenyi G, Cortez-Pinto H. Genetic polymorphisms of proangiogenic factors seem to favor hepatocellular carcinoma development in alcoholic cirrhosis. Eur J Gastroenterol Hepatol. 2014;26:438-43.

21. Ratnasari N, Nurdjanah S, Sadewa AH, Hakimi M. The role of vascular endothelial growth factor $-634 \mathrm{G} / \mathrm{C}$ and its soluble receptor on chronic liver disease and hepatocellular carcinoma. Arab J Gastroenterol. 2016;17:61-6.

22. Ratnasari N, Nurdjanah S, Sadewa AH, Hakimi M, Yano Y. Difference of polymorphism VEGF-gene rs699947 in Indonesian chronic liverdisease population. PLoS One. 2017:12:e0183503.

23. Wu LM, Xie HY, Zhou L, Yang Z, Zhang F, Zheng SS. A single nucleotide polymorphism in the vascular endothelial growth factor gene is associated with recurrence of hepatocellular carcinoma after transplantation. Arch Med Res. 2009:40:565-70.

24. Wu X, Xin Z, Zhang W, Wu J, Chen K, Wang H, Zhu X, Pan L, Li Z, Li H, Liu Y. Polymorphisms in the VEGFA promoter are associated with susceptibility to hepatocellular carcinoma by altering promoter activity. Int J Cancer. 2013; 133:1085-93.

25. Yvamoto EY, Ferreira RF, Nogueira V, Pinhe MA, Tenani GD, Andrade JG, Baitello ME, Gregório ML, Fucuta PS, Silva RF, Souza DR, Silva RC. Influence of vascular endothelial growth factor and alpha-feto protein on hepatocellular carcinoma. Genet Mol Res. 2015;14:17453-62.

26. Bakr NM. Awad A, A Moustafa E. Association of genetic variants in the interleukin-18 gene promoter with risk of hepatocellular carcinoma and metastasis in patients with hepatitis C virus infection. IUBMB Life. 2018;70: 165-74.

27. Bao J, Lu Y, Deng Y, Rong C, Liu Y, Huang X, Song L, Li S, Qin X. Association between IL-18 polymorphisms, serum levels, and HBV-related hepatocellular carcinoma in a Chinese population: a retrospective case-control study. Cancer Cell Int. 2015:15:72

28. Chen Q. The correlation between single nucleotide polymorphism of interleukin-18 gene promoter and genetic susceptibility to hepatocellular carcinoma. Immunol J. 2012; 28: 1051-1055. [Article in Chinese]

29. Dai ZJ, Liu XH, Wang M, Guo Y, Zhu W, Li X, Lin S, Tian T, Liu K, Zheng Y, Xu P, Jin T, Li X. IL-18 polymorphisms contribute to hepatitis B virus-related cirrhosis and hepatocellular carcinoma susceptibility in Chinese population: a case-control study. Oncotarget. 2017;8:81350-60.

30. Karra VK, Gumma PK, Chowdhury SJ, Ruttala R, Polipalli SK, Chakravarti A, Kar P. IL-18 polymorphisms in hepatitis B virus related liver disease. Cytokine. 2015;73:277-82

31. Kim YS, Cheong JY, Cho SW, Lee KM, Hwang JC, Oh B, Kimm K, Lee JA, Park BL, Cheong HS, Shin HD, Kim JH. A functional SNP of the Interleukin-18 gene is associated with the presence of hepatocellular carcinoma in hepatitis B virus-infected patients. Dig Dis Sci. 2009;54:2722-78.

32. Lau HK, Hsieh MJ, Yang SF, Wang HL, Kuo WH, Lee HL, Yeh CB. Association between Interleukin-18 Polymorphisms and Hepatocellular Carcinoma Occurrence and Clinical Progression. Int J Med Sci. 2016;13:556-61.

33. Migita K, Sawakami-Kobayashi K, Maeda Y, Nakao K, Kondoh S, Sugiura M, Kawasumi R, Segawa O, Tajima H, Machida M, Nakamura M, Yano K, Abiru S, Kawasaki E, Yatsuhashi H, Eguchi K, Ishibashi H. Interleukin-18 promoter polymorphisms and the disease progression of Hepatitis B virus-related liver disease. Transl Res. 2009;153:91-6.

34. Teixeira AC, Mendes CT Jr, Marano LA, Deghaide NH, Secaf M, Elias J Jr, Muglia V, Donadi EA, Martinelli AL. Alleles and genotypes of polymorphisms of IL-18, TNF-a and IFN- $\gamma$ are associated with a higher risk and severity of hepatocellular carcinoma (HCC) in Brazil. Hum Immunol. 2013;74:1024-9.

35. Zhang QX, Yao YQ, Li SL, Long Q. Association between interleukin-18 gene polymorphisms and hepatocellular carcinoma caused by hepatitis B virus. Chin J Hepatol. 2016; 24: 352. [Article in Chinese]

36. Eurich D, Boas-Knoop S, Morawietz L, Neuhaus R, Somasundaram R, Ruehl $M$, Neumann UP, Neuhaus P, Bahra M, Seehofer D. Association of mannosebinding lectin-2 gene polymorphism with the development of hepatitis Cinduced hepatocellular carcinoma. Liver Int. 2011;31:1006-12.

37. Gu X, Ji Q, Wang H, Jiang M, Yang J, Fang M, Wang M, Gao C. Genetic variants of mannose-binding lectin 2 gene influence progression and prognosis of patients with hepatitis B virus infection in China. Clin Res Hepatol Gastroenterol. 2016;40:614-21.

38. Lin Y, Su C, Niu J, Guo Z, Cai L. Impact of mannose-binding lectin 2 polymorphism on the risk of hepatocellular carcinoma: a case-control study in Chinese Han population. J Epidemiol. 2015;25:387-91.

39. Segat L, Fabris A, Padovan L, Milanese M, Pirulli D, Lupo F, Salizzoni M, Amoroso A, Crovella S. MBL2 and MASP2 gene polymorphisms in patients with hepatocellular carcinoma. J Viral Hepat. 2008;15:387-91.

40. Su C, Lin Y, Cai L, Mao Q, Niu J. Association between mannose-binding lectin variants, haplotypes and risk of hepatocellular carcinoma: A casecontrol study. Sci Rep. 2016;6:32147.

41. Moher D, Liberati A, Tetzlaff J, Altman DG, PRISMA group. Preferred reporting items for systematic reviews and meta-analyses: the PRISMA statement. Ann Intern Med. 2009:151:264-9.

42. Stang A. Critical evaluation of the Newcastle-Ottawa scale for the assessment of the quality of nonrandomized studies in meta-analyses. Eur J Epidemiol. 2010;25:603-5.

43. Rai V. Methylenetetrahydrofolate reductase (MTHFR) C677T Polymorphism and Alzheimer disease risk: a meta-analysis. Mol Neurobiol. 2017;54:1173-86.

44. Mostafa T, Taymour M. TNF-a -308 polymorphisms and male infertility risk: a meta-analysis and systematic review. J Adv Res. 2016;7:185-92.

45. Thompson SR, Humphries SE. Interleukin-18 genetics and inflammatory disease susceptibility. Genes Immun. 2007:8:91-9.

46. Luo J, Xu F, Lu GJ, Lin HC, Feng ZC. Low mannose-binding lectin (MBL) levels and MBL genetic polymorphisms associated with the risk of neonatal sepsis: an updated meta-analysis. Early Hum Dev. 2014;90:557-64.

47. Uitterlinden AG, Fang Y, Van Meurs JB, Pols HA, Van Leeuwen JP. Genetics and biology of vitamin D receptor polymorphisms. Gene. 2004;338:143-56.

48. Koukourakis MI, Papazoglou D, Giatromanolaki A, Bougioukas G, Maltezos E, Sivridis E. VEGF gene sequence variation defines VEGF gene expression 
status and angiogenic activity in non-small cell lung cancer. Lung Cancer. 2004:46:293-8.

49. Nishi A, Milner DA Jr, Giovannucci EL, Nishihara R, Tan AS, Kawachi I, Ogino S. Integration of molecular pathology, epidemiology and social science for global precision medicine. Expert Rev Mol Diagn. 2016;16:11-23.

50. Zhu SL, Zhao Y, Hu XY, Luo T, Chen ZS, Zhang Y, Yang SH, Zhou L, Li LQ. Genetic polymorphisms -137 (rs187238) and -607 (rs1946518) in the interleukin-18 promoter may not be associated with development of hepatocellular carcinoma. Sci Rep. 2016;6:39404.

51. Liu L, Guo W, Zhang J. Association of HLA-DRB1 gene polymorphisms with hepatocellular carcinoma risk: a meta-analysis. Minerva Med. 2017;108:176-84.

52. Dondeti MF, El-Maadawy EA, Talaat RM. Hepatitis-related hepatocellular carcinoma: Insights into cytokine gene polymorphisms. World J Gastroenterol. 2016;22:6800-16.

53. Lu SC, Zhong JH, Tan JT, Tang HL, Liu XG, Xiang BD, Li LQ, Peng T. Association between COX-2 gene polymorphisms and risk of hepatocellular carcinoma development: a meta-analysis. BMJ Open. 2015;5:e008263.

\section{Publisher's Note}

Springer Nature remains neutral with regard to jurisdictional claims in published maps and institutional affiliations.

Ready to submit your research? Choose BMC and benefit from:

- fast, convenient online submission

- thorough peer review by experienced researchers in your field

- rapid publication on acceptance

- support for research data, including large and complex data types

- gold Open Access which fosters wider collaboration and increased citations

- maximum visibility for your research: over $100 \mathrm{M}$ website views per year

At BMC, research is always in progress.

Learn more biomedcentral.com/submissions 